\title{
EVALUATION OF COMBINATION OF BIPHASIC CALCIUM PHOSPHATE AND PLATELET-RICH FIBRIN AS GRAFTING MATERIAL FOR SINUS LIFT AUGMENTATION
}

\author{
Aimen E. Khalfalla ${ }^{1} B D S$, Magued H. Fahmy ${ }^{2} P h D$, Adham A. El-Ashwah ${ }^{3} P h D$
}

\begin{abstract}
:
Introduction: The performance of implant surgery in the posterior maxilla often poses challenges due to insufficient available bone. Sinus floor elevation is developed to increase needed vertical height to overcome this problem. Many grafting materials had been used to augment subsinus area. Recently, several authors have shown that simultaneous sinus lift and implantation using autologous platelet-rich fibrin combined with biphasic calcium phosphate as a grafting material is a reliable procedure promoting bone augmentation in the maxillary sinus.

Objectives: This study was aiming to evaluate bone formation after maxillary sinus membrane elevation using lateral window technique and simultaneous implant placement using biphasic calcium phosphate combined with platelet rich fibrinas agrafting material.

Materials and methods: The present study was conducted on ten patients with age ranged from 30-50 years seeking implantation of their lost posterior maxillary teeth, and had limited bone height below the floor of the maxillary sinus, secondary to sinus pneumatization. Patients were selected on the basis of history, clinical examination and radiographic examination using panoramic radiography and cone-beam CT.

Conclusion: Platelet-rich fibrin in addition to biphasic calcium phosphate may favor the formation of new bone after maxillary sinus augmentation. The effectiveness of PRF depends not only on its features but also the properties of co-administered grafting material.
\end{abstract}

Key Words: Dental implant, Biphasic calcium phosphate, Platelet-rich fibrin, Sinus-lift.

1. B.D.S. Faculty of Dentistry, Tripoli University.

2. Professor of Oral \& Maxillofacial Surgery, Faculty of Dentistry, Alexandria University

3. Assistant Professor of Oral and Maxillofacial Surgery, Faculty of Dentistry, Alexandria University.

\section{INTRODUCTION}

Dental rehabilitation of edentulous posterior maxillary region is considered one of the most significant challenges that faces the Oral and Maxillofacial surgeon, as well as, the restorative dentist (1). Bone loss in the oral and maxillofacial region can result from trauma, congenital disorders, ablation of tumors and enucleation of cysts, anatomical events, such as pneumatization of the maxillary sinus, and infections (2). A solution to restore the lost oral function in edentulous patients is the placement of dental implants. However, a common problem for implant placement is the lack of sufficient bone height caused by excessive bone resorption, which occurs after tooth loss, particularly in the posterior maxillary bone (3).

Lateral Sinus Lift is one of the most widely used augmentation procedures. To increase vertically available bone volume of the edentulous posterior maxilla, where the bone often has poor quality and is reduced by the extended maxillary sinus. When considering that the minimum safe length of the implant is $10 \mathrm{~mm}$, the bone at the site of the first premolar is very low in one-fourth $(25 \%)$ of patients. The bone is insufficient in more than half of patients at the level of the second premolar, and in 80 to $90 \%$ of patients at the level of molars (4).

The most commonly used augmentation method for the sinus reconstruction was presented by Tatum in 1976 at
Alabama implant conference and published by Boyne and James in 1980. It intended to increase the vertical bone dimension in the posterior maxilla where access to the maxillary sinus is obtained by drilling a bony window in the lateral sinus wall while ensuring that the sinus membrane remains intact (5).

Various bone substitutes have been used for sinus floor elevation procedures. Autologous bone is considered the gold standard (6-8). However, the autologous bone transplanted may have an inconsistent rate of mineralization, due to the ratio of cortical to cancellous bone, and given that it also requires an additional donor-site surgery, it may not thus always be the material of choice for sinus floor elevation (8).

Platelet-rich fibrin (PRF) is an autologous fibrin matrix. It was described by Choukroun et al (9) specifically for oral and maxillofacial surgery applications. PRF can be classified as a second-generation platelet concentrate because it contains leukocytes and does not require an anticoagulant (10-12). Venous blood is collected in $10-\mathrm{mL}$ dry tubes and centrifuged for 12 minutes at $2700 \mathrm{rpm}$ $(\sim 400 \mathrm{~g})$. After centrifugation, 3 layers are obtained: red blood cells at the bottom, acellular plasma at the top (platelet-poor plasma), and PRF between the 2 layers. It was shown that, after centrifugation, $\sim 97 \%$ of platelets and $50 \%$ of leukocytes of the original blood volume were concentrated in the PRF (13). Previous studies showed the 
slow release of growth factors such as transforming growth factor $\beta 1$, platelet-derived growth factor $\beta$, and vascular endothelial growth factor, especially during the first 7 days $(14,15)$. Zumstein et al (16) also reported that this release continued with the decrease up to 28 days. PRF can be used as the sole biomaterial (17-29) or combined with different bone substitutes (18).The use of PRF in conjunction with grafting materials would accelerate bone regeneration.

Biphasic calcium phosphate (BCP) is a commonly used synthetic bone substitute comprising less soluble hydroxyapatite (HA) and more soluble $\beta$-tricalcium phosphate $(\beta$-TCP). It is a biocompatible, osteoconductive, and cost-effective biomaterial. The main advantage of BCP is that its chemical composition is similar to that of apatite in biological bone (19-21). The hard particles of HA support the bulk of the graft material, and $\beta$-TCP increases the replacement of its degradation products with blood vessels and lamellar bone (22).

Therefore, the present study was designed to evaluate the effect of PRF and BCP on bone regeneration in sinus lifting with simultaneous implant placement

\section{MATERIALS AND METHODS Selection of patients}

The present study was conducted on ten patients with age range from 30-50 years seeking implantation of their lost posterior maxillary teeth, and had limited bone height below the floor of the maxillary sinus, secondary to sinus pneumatization. They were selected from the Outpatient Clinic of oral and maxillofacial surgery department at the Faculty of Dentistry, Alexandria University. Patients were selected on the basis of history, clinical examination and radiographic examination using panoramic radiography and cone-beam CT to fit the following.

All patients were informed about sinus lifting and implant placement procedures and they gave their approval to participate in this study with written consent

\section{Inclusion criteria}

- No recent sinus surgery, severe sinus floor convolutions or prominent sinus septa.

- Acceptable inter-arch space for the future prosthesis.

- The ridge height at the site of implantation should be 4-7 $\mathrm{mm}$.

\section{Exclusion criteria}

Patients with immunologic diseases, unstable diabetes mellitus, maxillary sinus inflammations or other contraindicating systemic conditions were excluded.

\section{Material}

\section{A. Platelet Rich Fibrin (PRF)}

PRF clots and membranes were prepared as described by Choukroun et al. During surgery, $10 \mathrm{~mL}$ of venous blood was drawn in a glass-coated plastic tube without anticoagulant. A total $20 \mathrm{ml}$ of blood was taken from each patient and the tubes were immediately centrifuged at 2700 rpm for 12 minutes. Platelets were immediately activated, thus triggering a coagulation cascade.

After centrifugation, 3 layers were obtained: acellular plasma (platelet poor plasma) was concentrated at the top and was collected by syringe. Fibrin clots and red corpuscles were removed from the tube with a scalpel. PRF clot was immediately separated from red corpuscles by tweezers.

The PRF obtained were used in two ways:

- A part was cut into small pieces and mixed with graft material (BCP).

- $\quad$ The remaining part was pressed between 2 sterile compresses to be obtained as membrane.

\section{B. GENESIS-BCP [biphasic calcium phosphate] graft material}

The biphasic calcium phosphate that was used in the present thesis for maxillary sinus floor augmentation is based on a balance between a more stable phase $(\mathrm{HA}) \mathrm{Ca}^{10}\left(\mathrm{PO}^{4}\right)^{6}(\mathrm{OH})^{2}$ and a more soluble phase $(\beta-\mathrm{TCP})$ $\mathrm{Ca} 3\left(\mathrm{PO}^{4}\right)^{2}$ (Dio implant $-1464 \mathrm{u}$ - dong haeundae - gu, busan city. Korea).

Properties of BCP bioceramics relating to their medical applications include: macroporosity, microporosity, compressive strength, bioreactivity (associated with formation of carbonate hydroxyapatite on ceramic surfaces in vitro and in vivo), dissolution, and osteoconductivity. Biphasic calcium phosphate bioceramics is recommended for use as an alternative or additive to autogeneous bone for orthopedic and dental applications.

\section{The implant system}

Ten dentis (Dentis implant, DentisInc, Daegu, Korea) sclean tapered implants were used. This implant has an endosseos root form with resorbable blast media (RBM) surface treatment and tapered body with optimized thread, design and simplified conical prosthetic component.

It has an internal hex connection and having different lengths $(8,10,12,14 \mathrm{~mm})$ and diameter $(3.7,4.1,4.3,4.7 \mathrm{~mm})$.

\section{Methods}

\section{Preoperative phase}

\section{A. Clinical examination}

Patients' data were collected; name, gender and age, medical and dental history were taken. All patients were subjected to extra oral examination; lymph node examination and examination of the area above the maxillary sinus for the presence of any tenderness or swelling. Also, intra-oral examination to determine the condition of the edentulous maxillary area and the opposing dentition. The oral mucosa of the edentulous area was examined for color, texture, firmness and thickness, the buccal vestibule and the palate were examined for the presence of any pathologic condition.

\section{B. Radiographic examination}

Orthopantomograms were performed for all patients for pre-operative preliminary assessment.

Cone beam Computerized Tomography (CBCT) were performed for all patients preoperatively to measure the vertical height of the bone between the alveolar crest and the floor of the maxillary sinus, and the bone width at the selected edentulous area to select the suitable implant site, length and width (Figure1). 


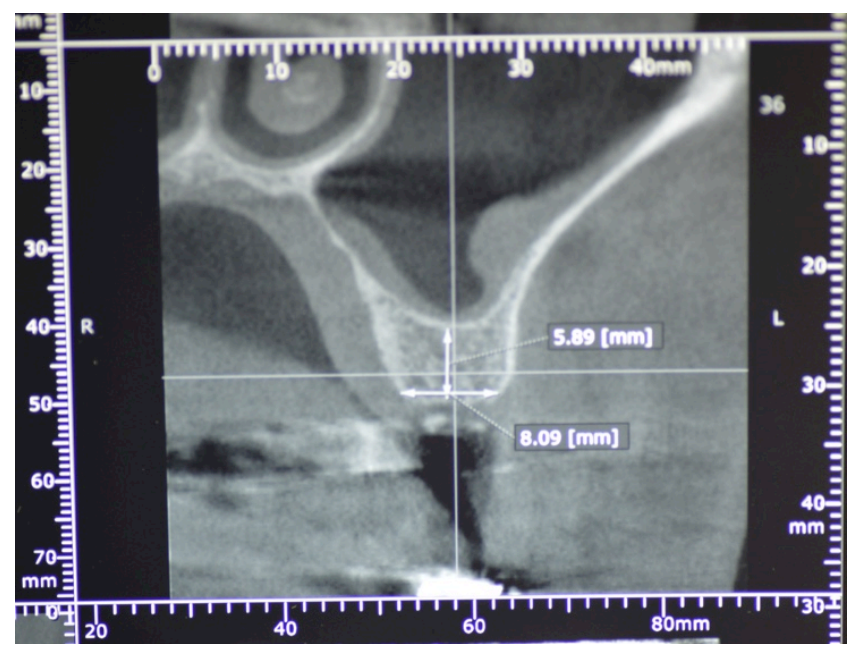

Fig. 1: A photoradiograph showing preoperative cone beam C.T.

\section{Surgical phase}

A modified Caldwell-Luc sinus augmentation was performed under local anesthesia (2\% lidocaine hydrochloride-epinephrine 1:100,000; Huons Co., Seoul, Korea) was achieved by maxillary vestibular infiltration and middle/posterior superior alveolar nerve block.

A trapezoid flap was used to fully expose the alveolar ridge and the lateral wall of the maxillary Sinus via elevation of a full thickness mucoperiosteal flap, an incision was made, 2 to $3 \mathrm{~mm}$ on the palatal side of the crest of the ridge with two vertical releasing incision at least $15 \mathrm{~mm}$ mesial to the antral opening.

Bony window, round to elliptical in shape, was cut $5 \mathrm{~mm}$ away from the crest of the ridge. (Figure 2) With using a diamond round bur and piezoelectric device (Surgybone Silfradent, srl, Sofia, Italy) and the antral mucosa was then carefully elevated and the prepared antrostomy was subsequently in-fractured, like a trap-door, and used as the superior border of the sinus compartment, leaving it attached to the underlying Schneiderian membrane eand being careful not to create perforations. The sequence of membrane elevation was used from the sinus floor, toward the posterior wall, then superior wall and finally to the anterior wall.

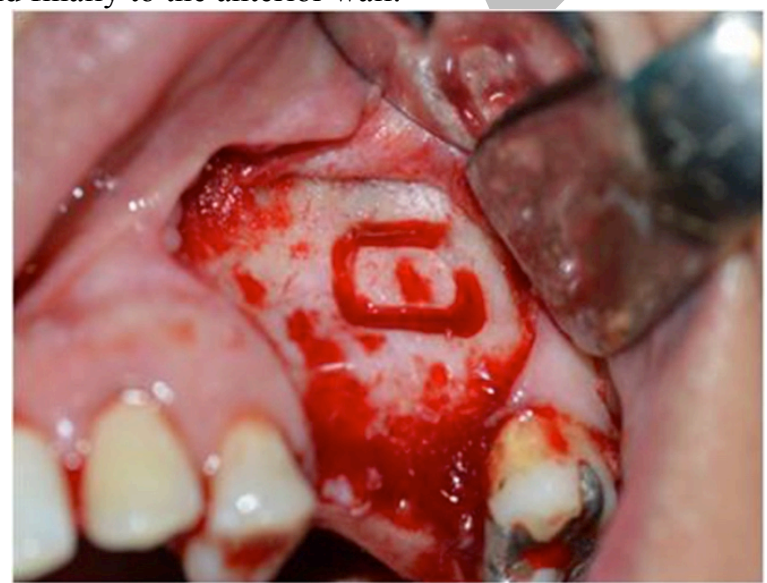

Fig. 2: A photograph showing the outline of the window to be opened on lateral wall of maxillary sinus.
The membrane was elevated from the medial sinus wall to allow for optimal graft placement.

When the sinus membrane was intact, a bellows effect was observed as the patient breathed. In the event of an accidental perforation, a PRF as membrane was used to seal the communication between the graft and the sinus cavity.

Drilling at the implant site was performed using the implant micromotor and Dentis implant drilling kit at low speed (800to $1200 \mathrm{rpm})$ high torque $(35 \mathrm{Ncm})$ along with copious irrigation (external and internal) of normal saline to prevent thermal injury to the bone. Tapered design implants (Dentis implant, DentisInc, Daegu, Korea) were placed in the prepared site and then the osteotomized segment was supported on the implant head. (Figure 3)

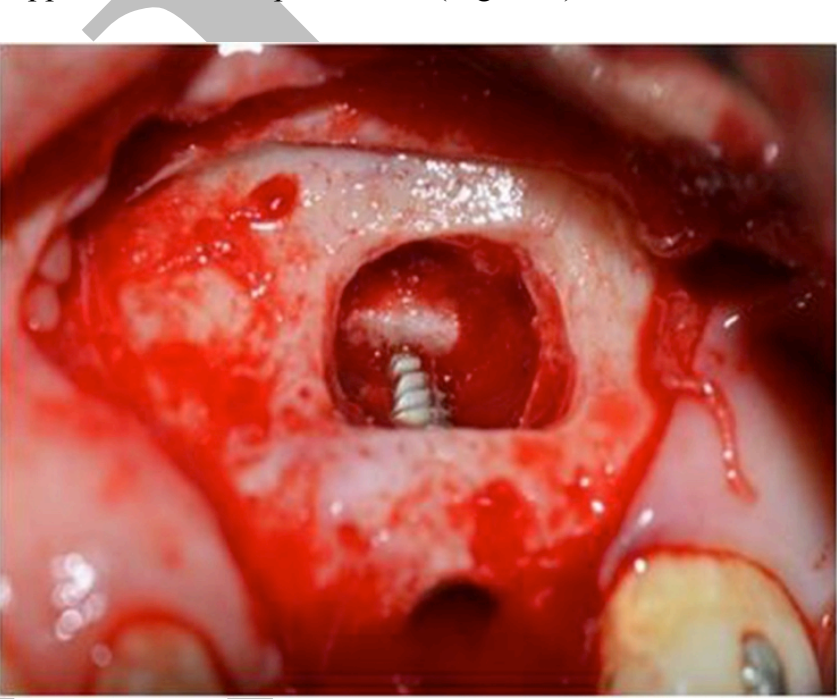

Fig. 3: A photograph showing the implant placed in the prepared bed.

The space created was filled with mixed BCP and PRF and the implant surface was covered with the same. (Figure 4)

The lateral window was then covered with PRF membrane to avoid the fibrous adhesion between the inner surface of the flap and bone graft. (Figure 5) After the placement of the membrane, the mucoperiosteal flap was replaced and sutured by simple interrupted suture technique, using black silk suture 3/0 (Ethicon SETA, B1130 Brussels, Belgium).

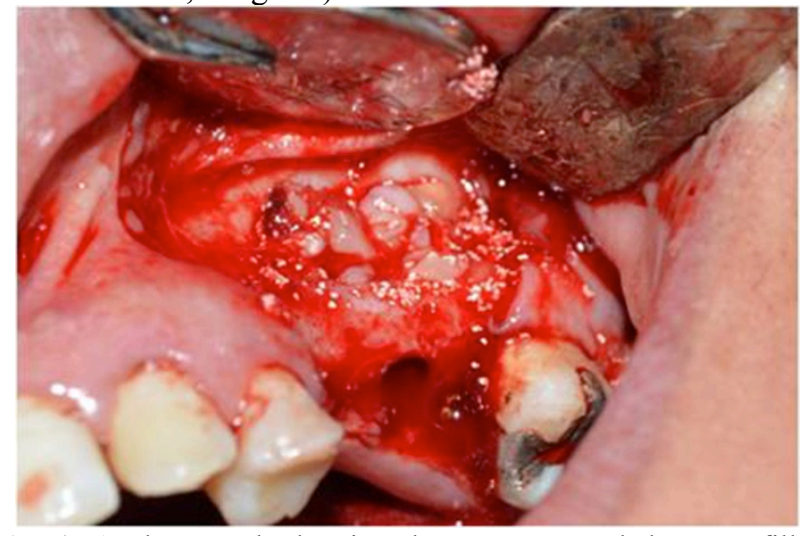

Fig. 4: A photograph showing the space created that was filled with mixed BCP and PRF. 


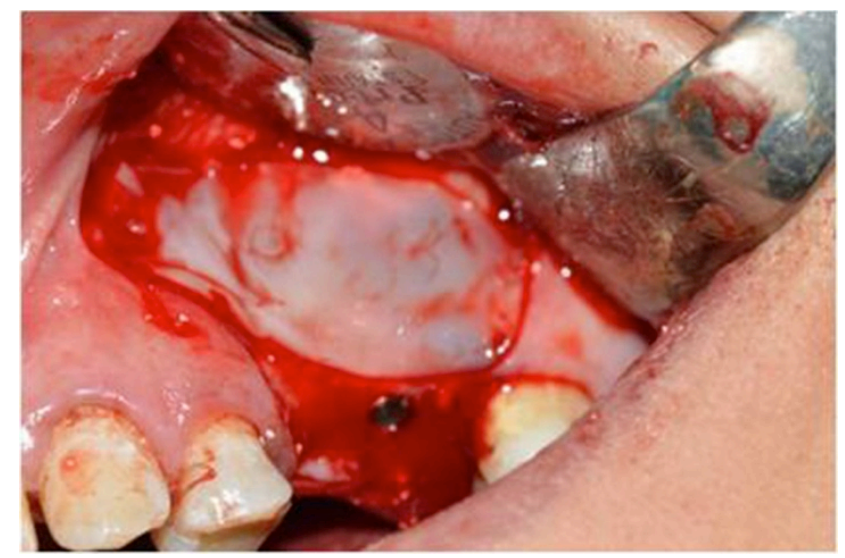

Fig. 5: Photograph showing the lateral window covered with PRF membrane.

\section{Post-operative phase}

\section{A. Postoperative Instructions}

- The patients were instructed to apply cold fomentations and avoid hot drinks for the first day.

- Apply hot fomentations from the second day.

- Avoid chewing hard food at implant site.

- Oral hygiene recommendations by the use of regular antiseptic mouthwash and soft bristle toothbrush.

\section{B. Postoperative medications}

1. Broad spectrum antibiotic; Amoxicillin 1g, Clavulanic acid $125 \mathrm{mg}$ tablets (Augmentin $1 \mathrm{gm}$ Smithline Beecham Pharmaceutical Co., Bentford, England) in combination with metronidazole (Amrizole $500 \mathrm{mg}$ tablets, Amriya Pharmaceutical Industries, Egypt) $500 \mathrm{mg}$ tablets twice daily for 5 days to avoid post-operative infection.

2. Non-steroidal anti-inflammatory analgesic in the form of diclofenac potassium (Cataflam 50mg tablets, Novartis PharmaAG,Basle, Switzerland )50mg tablets 3 times daily for 7-10 days to avoid the possibility of inflammation, edema and pain.

3. Chlorhexidine HCL $0.12 \%$ (Hexitol Mouth wash,Thearab drug Co., Cairo, Egypt) Mouth wash three times daily starting on the 2 nd postoperative day for 10 days.

4. Ephidrine (Otrivin spray/nasal Drops $10 \mathrm{ml}$, Novartis Pharma AG, Basle, Switzerland )nasal drops 3-5 times daily for 5 days.

\section{Follow up phase}

\section{A. Clinical examination}

All patients were examined the day after surgery then weekly for the first month postoperatively, then on an interval of 3, 6 and 9 months postoperatively. The clinical parameters of importance for determination of implant success includes:

The presence of pain, tenderness, discomfort, wound dehiscence, implant mobility or any other complications related to the sinus lifting procedure or implant placement.

\section{Pain and discomfort}

Pain was examined using visual analogue scale (VAS). The VAS is a simple, uni-dimensional measure of pain intensity that allows the severity of the pain experienced to be expressed as a numeric value. It is presented as a plain horizontal $10 \mathrm{~cm}$ line. The patients were instructed to bisect the line at a point appropriate to their present discomfort. A zero value regarded to be pain free; whereas the most severe pain was rated at 10 .

\section{Wound dehiscence}

Wound dehiscence was evaluated through inspection of the wound area regarding its presence or absence.

\section{Nasal obstruction}

It was evaluated by asking the patient during the follow up phase regarding its presence of absence.

\section{Edema}

It was evaluated subjectively by inspection.

\section{Evaluation of implant mobility}

Evaluation of implant mobility as described by Smith and Zarb. It was carried out by the application of a back and forth pressure with two instrument handles then simple grading was recorded as $(0=$ no mobility noted $),(1=$ mobility noted) at 6 month.

\section{A. Radiographic evaluation}

- Immediate post-operative panoramic x-rays were taken to evaluate the position and angulation of the implants, then at 3 and 6 post-operative months to detect any radiographic changes around the implants.

- Cone-beam CT was preformed for all patients at 9 post -operative months after implant insertion to assess bone formation and density (Figure 6). CBCT measurements were performed using On Diamond 3DApp-DBM.

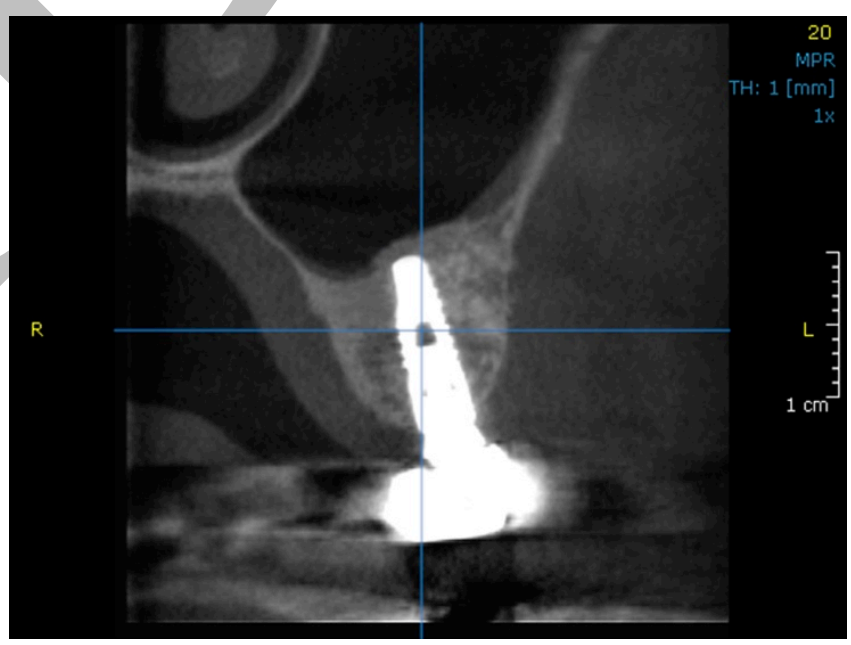

Fig. 6: A photoradiograph showing 9 months cone beam C.T

\section{Prosthetic phase}

Definitive porcelain fused to metal restorations were delivered to all patients at the $6^{\text {th }}$ postoperative month.

\section{Statistical analysis}

Data were presented as mean and standard deviation (SD) values. Paired-test was used to compare between preoperative and postoperative bone height. Wilcoxon 
signed ranks-test was used to compare between preoperative and postoperative bone density. The significance level was set at $\mathrm{P} \leq 0.05$.

\section{RESULTS}

In this study, ten sinus floor augmentations were performed on ten patients. The selected patients were 4 males and 6 females, and their age ranged from 30-50 years. The mean height of the alveolar ridge from the marginal crest to floor of the maxillary sinus was $5.69 \mathrm{~mm} \pm 0.95 \mathrm{~mm}$ (Range: $4.49-6.8 \mathrm{~mm})$.

Ten implants were placed in the maxillary posterior teeth, 10 at the molar region. The patients received implants with length ranged from $10.0-12.0 \mathrm{~mm}$. All the placed implants were $3.7 \mathrm{~mm}$ in diameter.

\section{1-Pain index:}

Pain index recorded its highest ranging from 1-3 with a mean of $(2.0 \pm 0.82)$ during the 1 st post-operative day, $0-2$ with a mean of $(1.00 \pm 0.82)$ during the 1st post-operative week, $0-1$ with a mean of $(0.29 \pm 0.49)$ during the 2 nd postoperative week. While no pain was recorded since the $3^{\text {rd }}$ week postoperatively till the rest of the follow up period. It was statistically significant starting from the 1 st week postoperatively compared to the 1 st post-operative day $(\mathrm{p} \leq$ $0.05)$.

\section{2- Tenderness}

Tenderness was present only in 3 patients during the $1^{\text {st }}$ post-operative day and absent in all patients during the rest of the follow up period. Swelling was observed in 4 patients during the 1st postoperative day, and resolved completely in all cases during the 1st post-operative week.

\section{3-Nasal obstruction and nasal bleeding}

Nasal obstruction was a complaint for 4 patients during the 1st postoperative day. Only 3 patients had complained during the 1st and 2nd postoperative weeks. Nasal bleeding was absent in all cases.

\section{4- Wound dehiscence}

No wound dehiscence was present post operatively in all of the cases.

\section{5- Edema}

Minimal edema was present post operatively in all of the cases.

\section{6- Implant mobility}

All implants was stable in all follow up period and during abutment tightening.

\section{7- Radiographic evaluation}

Cone-beam CT9 months postoperatively showed statistically significant increase in bone height and density. The mean postoperative bone height measured from the floor of the maxillary sinus and alveolar crest was (10.67 \pm $1.38 \mathrm{~mm}$ ) with a range from $8.20-12.0 \mathrm{~mm}$. A significant change was indicated compared to the preoperative bone height, with a mean percentage of change of $90.72 \%$, as shown in (table 1).
Table 1: Comparison between preoperative and postoperative bone height.

\begin{tabular}{|l|c|c|c|c||}
\hline \multirow{2}{*}{} & \multicolumn{2}{|c|}{ Bone height } & \multirow{2}{*}{ t } & \multirow{2}{*}{ p } \\
\cline { 2 - 3 } & Preoperative & Postoperative & & \\
\hline Min. - Max. & $4.0-6.90$ & $8.20-12.0$ & & \\
Mean \pm SD. & $5.69 \pm 0.95$ & $10.67 \pm 1.38$ & $12.630^{*}$ & $<0.001^{*}$ \\
Median & 5.72 & 11.10 & & \\
\hline Change & \multicolumn{2}{|c|}{$\mathbf{4 . 9 8} \pm \mathbf{1 . 2 5}$} & & \\
\hline \% of change & \multicolumn{2}{|c|}{$\mathbf{9 0 . 7 2 \%}$} & & \\
\hline
\end{tabular}

The mean postoperative bone density around the implants after 9 month was $(994.82 \pm 113.11 \mathrm{HU})$ and ranged from (832.70-1117.0 HU), which changed significantly compared to the preoperative bone density, with a mean percentage of change $310.39 \%$, as shown in (table 2 ).

Table 2: Comparison between preoperative and postoperative bone density.

\begin{tabular}{|c|c|c|c|c|}
\hline & \multicolumn{2}{|c|}{ Bone density } & \multirow{2}{*}{$\mathbf{Z}$} & \multirow{2}{*}{$\mathbf{p}$} \\
\hline & Preoperative & Postoperative & & \\
\hline Min. - Max. & $-536.33--402.15$ & $832.70-1117.0$ & & \\
\hline Mean $\pm \mathrm{SD}$. & $-474.26 \pm 60.85$ & $994.82 \pm 113.11$ & $2.803^{*}$ & $0.005^{*}$ \\
\hline Median & -506.50 & 1026.71 & & \\
\hline Change & \multicolumn{2}{|c|}{$1469.08 \pm 170.55$} & & \\
\hline$\%$ of change & \multicolumn{2}{|c|}{$310.39 \%$} & & \\
\hline
\end{tabular}

The lateral-posterior parts of the edentulous upper jaw often have anatomical limitations, which result in a continuous challenge for the dental surgeon. In these areas, in fact, the primary stability of implants and the elusion of intra- and post-operative complications may be invalidated by an inadequate three-dimensional bone support, that is to say an insufficient height and/or thickness of the alveolar crest. This bone deficiency may result from an excessive pneumatization of the maxillary sinus (large sinus cavities), from an important atrophy of the alveolar crest referable to dental extractions and/or periodontal diseases or from both of these causes (23-25). Besides, the reabsorption process in edentulous posterior maxillary regions can determine insufficient vertical dimensions for implant positioning often requiring additional surgical procedures.

The choice of the lateral window technique in this study was to allow proper visualization of Schneiderian membrane and allows proper and easier application of the PRF mixed with BCP For simultaneous implant placement with sinus floor elevation procedure, a minimum of $4 \mathrm{~mm}$ of residual bone height has been recommended in order to achieve sufficient initial implant stability $(26,27)$.

Many types of materials are used in sinus lift: autologous bone, demineralized and freeze- dried bone, hydroxyapatite and different combinations of these materials, often with 
satisfactory results in terms of biocompatibility, induction of bone formation and, of course, implant stability $(28,29)$. Among the variety of grafting materials, PRF has become a focus of current studies due to its potential to accelerate and improve the healing process. In the present study, we evaluated PRF in combination with BCP for sinus augmentation (18).

Each bone substitute has advantages and disadvantages. Autogenous bone grafts are still considered to be the gold standard because of their osteogenic, osteoconductive, and osteoinductive properties. Nevertheless, this type of augmentation has the disadvantages of requiring for a second operative donor site and having rapid resorption. Allogenic and xenografts grafts are produced from other humans or animals, making these materials unacceptable to some patients. These materials also involve the risk of carrying disease. Synthetic or alloplastic grafting products such as HA/TCP composite ceramics (BCP) therefore provide another option. The advantages of BCP compared with autogenous grafts are their synthetic origin, biocompatibility, osteoconductivity, unlimited quantity, and avoidance of a second surgical site. Therefore, clinically applied BCP was preferred in the study (20).

The literature includes few studies using only PRF or graft materials with different characteristics combined with PRF.

PRF was used as graft material in this study because it is a fibrin-matrix in which platelet, cytokines and cells are entrapped and released after a certain time and can serve as a resorbable membrane. It is considered to be a healthy biomaterial, and it was initially used in oral implantology by its promoters, and presently, its application has been advocated in various disciplines of dentistry (30).

In the present study no wound dehiscence was present post operatively in any of the cases and radiographic evaluation by CBCT 9 months postoperatively revealed the absence of any fluid level or inflammatory process.

Nasal bleeding was absent in all cases postoperatively during the follow up period. While, pain and discomfort scale significantly changed after the first day. Swelling was present in 3 cases on the first day only and nasal obstruction was a postoperative complain in 3 cases on the first day, with its absence in all cases for the rest of the follow up period. This coincides with Carini et al (31) in 2014, in their study where they observed better healing quality by reducing patient's postoperative morbidity when using piezosurgery.

Also, in agreement with Dohan et al (32) in 2006, where they mentioned that PRF might decrease many harmful effects due to inflammatory processes that are inherent to the surgical act itself, mainly by correcting certain destructive and noxious excesses during the healing process of wounded tissues.

In addition, He et al (33) in 2009 in an in vitro study reported the superiority of PRF in the expression of alkaline phosphatase and induction of mineralization, caused markedly by release of TGF- $\beta 1$ and PDGF-AB. They remarked that $\mathrm{PRF}$ released autologous growth factors gradually and expressed stronger and more durable effect on proliferation and differentiation of osteoblasts than PRP.

In the present study, CBCT obtained 9 months after surgery revealed sufficient newly formed bone in all treated cases, elevating the sinus membrane showing repneumatization around the implants apices. The new bone showed adaptation in shape and volume with the repneumatized maxillary sinus with a decreased noted visibility of the original sinus floor. The apex of the implants remained surrounded with bone radiographically. This indicated the stability of the newly formed bone and bone maturation. The sinus membrane is maintained elevated and the bone gained is preserved.

This is in agreement with a 6 year follow up study by Simonpieri et al (34) in 2011, where they performed lateral sinus lifting on 20 patients with simultaneous implant placement and PRF was used to fill the subsinus cavity. They observed that the final level of the new sinus floor was always in continuation with the implant apical end.

In this study, there was a significant increase in bone height at the end of the procedure at 9 month follow up period. Because the implants served as a tent to maintain the height of the bone healing space the final vertical bone height was dependent on the implant length.

In addition, the density of the new bone formed around the implants was significant at 9 post-operative months.

This is in agreement with a study conducted by Mazor et al (35) in 2009, which was designed for the validation of the PRF as a filling material in 25 sinus elevations performedon20patients. Radiographic analysis showed that the final bone gain was always significant with the implant length.

In the current study implant loading was performed at 6 months postoperatively this is following a study that was conducted by Kim et al (36) to compare the survival rate and surrounding tissue condition of sinus bone grafts with simultaneous implant placement between 4-month and 6month occlusal loading after implantation, and concluded that the cases in which the residual bone was $>3 \mathrm{~mm}$ and primary implant stability could be obtained loading is possible 4 months after the sinus bone graft and simultaneous implant placement with no significance compared to 6 month loading in implant stability.

In the current study, there was a significant change in the pain visual analogue scale with minimal edema and tenderness through the follow up phase.

\section{CONCLUSIONS}

From the results of this study we can conclude that, PRF in addition to BCP may favor the formation of new bone after maxillary sinus augmentation. The effectiveness of PRF depends not only on its features but also the properties of co-administered grafting material. Although it is reported that PRF dissolves more slowly than other platelet concentrates, it does not exceed months when the clinical samples only can be collected; therefore, further studies are necessary to investigate the effects of PRF and coadministered biomaterials on bone formation. 


\section{CONFLICT OF INTEREST}

The authors declare that they have no conflicts of interest.

\section{REFERENCES}

1. Raja SV. Management of the posterior maxilla with sinus Lift: Review of Techniques. J Oral MaxillofacSurg 2009; 67: 1730.

2. Diaz-Romeral-Bautista M, Manchon-Miralles A, Asenjo-Cabezon J, Cebrian-Carretero JL, TorresGarcia-DencheJ, Linares-Garcia-Valdecasas R. Autogenous calvarium bone grafting as a treatment for severe bone resorption in the upper maxilla: a case report. Med Oral Patol Oral Cir Bucal 2010; 15:e361-5.

3. Hieu PD, Chung JH, Yim SB, Hong KS. A radiographical study on the changes in height of grafting materials after sinus lift: a comparison between two types of xenogenic materials. J Periodontol Implant Sci 2010; 40: 25-32.

4. Browaeys H, BouvryP, De Bruyn H. A literature review on biomaterial in sinus augmentation procedures. Clin implant Dent Relat Res 2007;9:166177.

5. Boyne PJ, James RA. Grafting of the maxillary sinus floor with autogenous marrow and bone. J Oral Surg 1980; 38: 613-6.

6. Klijn RJ, Meijer GJ, Bronkhorst EM, Jansen JA. A meta-analysis of histomorphometric results and graft healing time of various biomaterials compared to autologous bone used as sinus floor augmentation material in humans. Tissue Eng Part B Rev 2010;16:493-507.

7. Klijn RJ, Meijer GJ, Bronkhorst EM, Jansen JA. Sinus floor augmentation surgery using autologous bone grafts from various donor sites: a meta-analysis of the total bone volume. Tissue Eng Part B Rev 2010;16:295-303.

8. Schlegel KA, Schultze-Mosgau S, Wiltfang J, Neukam FW, Rupprecht S, Thorwarth M. Changes of mineralization of free autogenous bone grafts used for sinus floor elevation. Clin Oral Implants Res 2006;17:673-8.

9. Choukroun J, Adda F, Schoeffler C. An opportunity in perioimplantology: The PRF. Implantodontie 2001;42:55-62.

10. Dohan Ehrenfest D.M., Rasmusson L., Albrektsson T. Classification of platelet concentrates: from pure platelet-rich plasma (P-PRP) to leucocyte- and plateletrich fibrin (L-PRF). Trends Biotechnol2009;27:158-67.

11. Dohan DM, Choukroun J, Diss A. Platelet-rich fibrin (PRF): a second-generation platelet concentrate. Part III: leucocyte activation: a new feature for platelet concentrates? Oral Surg Oral Med Oral Pathol Oral Radiol Endod 2006;101: 51-5.

12. Dohan DM, Choukroun J, Diss A. Platelet-rich fibrin (PRF): a second-generation platelet concentrate. Part II: platelet-related biologic features. Oral Surg Oral Med Oral Pathol Oral Radiol Endod 2006;101: 45-50.
13. Dohan Ehrenfest DM, Del Corso M, Diss A. Threedimensional architecture and cell composition of a Choukroun's platelet-rich fibrin clot and membrane. J Periodontol 2010;81:546-55.

14. Dohan Ehrenfest DM, Bielecki T, Jimbo R. Do the fibrin architecture and leukocyte content influence the growth factor release of platelet concentrates? An evidence-based answer comparing a pure platelet-rich plasma (P-PRP) gel and a leukocyte- and platelet-rich fibrin (L-PRF). Curr Pharm Biotechnol 2012;13:114552.

15. Dohan Ehrenfest DM, de Peppo GM, Doglioli P. Slow release of growth factors and thrombospondin-1 in Choukroun's platelet-rich fibrin (PRF): a gold standard to achieve for all surgical platelet concentrates technologies. Growth Factors 2009;27:63-9.

16. Zumstein MA, Berger S, Schober M. Leukocyte- and platelet-rich fibrin (L-PRF) for long-term delivery of growth factor in rotator cuff repair: review, preliminary results and future directions. Curr Pharm Biotechnol 2012;13:1196-206.

17. Ozdemir H, Ezirganli S, Isa Kara M. Effects of platelet rich fibrin alone used with rigid titanium barrier. Arch Oral Biol 2013;58:537-44.

18. Bajaj P, Pradeep AR, Agarwal E, Rao NS, Naik SB, Priyanka $\mathrm{N}$, et al. Comparative evaluation of autologous platelet-rich fibrin and platelet-rich plasma in the treatment of three-wall intrabony defects in chronic periodontitis: a randomized controlled clinical trial. J Periodontol 2013; 8: 573-81.

19. Nieminen T, Rantala I, Hiidenheimo I. Degradative and mechanical properties of a novel resorbable plating system during a 3-year follow-up in vivo and in vitro. $\mathbf{J}$ Mater Sci Mater Med 2008;19:1155-63.

20. Albrektsson $\mathrm{T}$, Johansson C. Osteoinduction, osteoconduction and osseointegration. Eur Spine J 2001;10: 96-101.

21. Constantz BR, Barr BM, Ison IC. Histological, chemical, and crystallographic analysis of four calcium phosphate cements in different rabbit osseous sites. J Biomed Mater Res 1998;43:451-61.

22. Almasri M, Altalibi M. Efficacy of reconstruction of alveolar bone using an alloplastic hydroxyapatite tricalcium phosphate graft under biodegradable chambers. Br J Oral Maxillofac Surg 2011;49:469-73.

23. Reinert $S$, König $S$, Bremerich A, Eufinger $H$, Krimmel M. Stability of bone grafting and placement of implants in the severely atrophic maxilla. Br J Oral Maxillofac Surg 2003; 41: 249-55.

24. Scarano A, Degidi M, Iezzi G, Pecora G, Piattelli M, Orsini G, et al. Maxillary sinus augmentation with different bio- materials: a comparative histologic and histomor- phometric study in man. Implant Dent 2006; 15: 197-207

25. Sakka S, Coulthard P. Bone quality: a reality for the process of osseointegration. Implant Dent 2009; 18:480-5.

26. Felice P, Pistilli R, Piattelli M, Soardi E, Barausse C, 
Esposito M. 1-stage versus 2 stage lateral sinus lift procedures: 1-year post-loading results of a multicenter randomised controlled trial. Eur J Oral Implantol2014;7:65-75.

27. Pariente L, Dada K, Daas M. Mini-lateral windows for minimally invasive maxillary sinus augmentation: case series of a new technique. Implant Dent 2014;23: 3717.

28. Galindo-moreno P, Moreno-riestra I, Avila G, Fernández-barbero JE, Mesa F, Aguilar M, et al. Histomorphometric comparison of maxillar y pristine bone and composite bone graft biopsies obtained after sinus augmentation. Clin Oral Implants Res 2010; 21: 122-8.

29. Scarano A, Degidi M, Iezzi G, Pecora G, Piattelli M, Orsini G, et al. Maxillary sinus augmentation with different bio- materials: a comparative histologic and histomorphometric study in man. Implant Dent 2006; 15:197-207.

30. Dohan DM, Choukroun J, Diss A, Dohan SL, Dohan AJ, Mouhyi J et al. Platelet-rich fibrin (PRF): a second generation platelet concentrate. Part II: platelet-related biologic features. Oral Surg Oral Med Oral Pathol Oral Radiol Endod 2006;101:e45-50.

31. Pereira CC, Gealh WC, Meorin-Nogueira L, GarciaJúnior IR, Okamoto R. Piezosurgery applied to implant dentistry: clinical and biological aspects. J Oral Implantol 2014;40:401-8.

32. Dohan DM, Choukroun J, Diss A, Dohan SL, Dohan AJ, Mouhyi J, et al. Platelet-rich fibrin (PRF): a second generation platelet concentrate. Part III: leucocyte activation: a new feature for platelet concentrate?. Oral Surg Oral Med Oral Pathol Oral Radiol Endod 2006; 101: 51-5.

33. He L, Lin $\mathrm{Y}, \mathrm{Hu} \mathrm{X}$, Zhang $\mathrm{Y}, \mathrm{Wu} \mathrm{H}$. A comparative study of platelet-rich fibrin (PRF) and platelet-rich plasma (PRP) on the effect of proliferation and differentiation of rat osteoblasts in vitro. Oral Surg Oral Med Oral Pathol Oral Radiol Endod 2009;108:707-13.

34. Simonpieri A, Choukroun J, Del Corso M, SammartinoG, DohanEhrenfest DM. Simultaneous sinus-lift and implantation using microthreaded implants and leukocyte- and platelet-rich fibrin as sole grafting material: a six-year experience. Implant Dent 2011;20:2-12.

35. Mazor Z, Horowitz RA, Del Corso M, Prasad HS, Rohrer MD, Dohan Ehrenfest DM. Sinus floor augmentation with simultaneous implant placement using Choukroun's platelet-rich fibrin as the sole grafting material: a radiologic and histologic study at 6 months. J Periodontol 2009;80:2056-64.

36. Kim BJ, Lee JH. The retrospective study of survival rate of implants with maxillary sinus floor elevation. $\mathbf{J}$ Korean Assoc Oral Maxillofac Surg 2010;36:108-18. 